\title{
TERMOS DA LINGUAGEM DE ENFERMAGEM IDENTIFICADOS EM REGISTROS DE UMA UTI NEONATAL*
}

\author{
TERMS OF THE NURSING LANGUAGE IDENTIFIED IN REGISTERS OF AN UIT NEONATAL*
}

TÉRMINOS DE LA LENGUAJE DE ENFERMERIA IDENTIFICADOS EN REGISTROS DE UNA UTI NEONATAL*

\section{Candice Cavalcanti de Alburquerque ${ }^{1}$, Maria Miriam Lima da Nóbrega ${ }^{2}$, Telma Ribeiro Garcia ${ }^{3}$}

RESUMO: A avaliação do registro de enfermagem sempre foi uma parte essencial do desenvolvimento profissional, porém, ainda não é possível se descrever o conteúdo da prática, pois a linguagem utilizada no cuidado não se tornou suficientemente unívoca. Nessa perspectiva percebe-se a relevância da identificação de uma linguagem específica utilizada pelos enfermeiros, para o registro de suas atividades. Este estudo objetivou identificar os termos empregados pelos componentes da equipe de enfermagem, nos registros de prontuários da Unidade de Terapia Intensiva Neonatal (UTIN) de um hospital escola; e fazer o mapeamento cruzado dos termos identificados com os constantes na CIPE $^{\circledR}$ Versão 1. Utilizou-se o método retrospectivo na coleta de dados. Na extração e normalização de termos, chegou-se a 161 termos constantes nos sete eixos da CIPE $^{\circledR} 1$, e a 603 não constantes. Analisando os 161 termos constantes, encontrou-se 45 termos pertencentes ao eixo Foco, 8 ao eixo Julgamento, 23 ao eixo Meios, 39 ao eixo Ação, 12 ao eixo Tempo, 30 ao eixo Localização, e 4 ao eixo Cliente, os quais foram analisados segundo seu índice de relevância para a UTIN. De acordo com os resultados obtidos, pode-se concluir que os objetivos que nortearam esta pesquisa foram alcançados e a trajetória metodológica mostrou-se adequada. Considera-se 0 estudo importante para 0 conhecimento do vocabulário utilizado na UTIN, contribuindo para a construção de uma nomenclatura específica para esta unidade.

PALAVRAS CHAVE: Enfermagem; Linguagem; Registros de Enfermagem, Terminologia.

ABSTRACT: The evaluation of the nursing records was always an essential part of the professional development; however, it is not still possible to describe the content of the practice, because the language used in the care didn't become sufficiently univocal. In that perspective it is noticed the relevance of the identification of a specific language used by the male nurses, for the registration of your activities. This study aimed to identify the terms used by the components of the nursing team, in the nursing records of the Unit of Intensive Therapy Neonatal (UITN) of a hospital school; and to cross map the identified terms with the constants in the seven axes of CIPE ${ }^{\circledR}$ Version 1 . In the collection of data it was used the retrospective method. After the extraction and normalization of terms, it was found 161 terms constants in the seven axes of CIPE $® 1$, and 603 not constants. Analyzing the 166 constants, observed 45 in the axis Focus, 8 in the axis Judgment, 23 in the axis Means, 39 in the axis Action, 12 in the axis Time, 30 in the axis Location, and 4 in the axis Client, which were analyzed to verify their relevance for UITN. According to the results, it can be concluded that the objectives of this research were reached and that the methodological paths were appropriate. This study is considered of great importance to the knowledge of the vocabulary used in UITN, contributing for the construction of a specific nomenclature for this unit.

KEY WORDS: Nursing; Language; Nursing records, Terminology.

RESUMEN: La evaluación del registro de enfermería siempre era una parte esencial del desarrollo profesional, sin embargo, todavía no es posible describir el volumen de la práctica, porque el idioma usó en el cuidado no se vuelva los unívoca suficientemente. En esa perspectiva se nota la relevancia de la identificación de un idioma específico usada por las enfermeras, para el registro de sus actividades. Este estudio intentó identificar los términos usados por los componentes del equipo de enfermería, en los registros de la Unidad de Terapia Intensiva Neonatal (UTIN) de un hospital escuela; y hacer lo cross-mapping de los términos identificados con los términos constantes en la CIPE ${ }^{\circledR}$ Versión 1. En la colección de datos se utilizó el método retrospectivo. En la extracción y normalización de los términos, se llegó a 161 términos constantes en los eje de la CIPE ${ }^{\circledR} 1$, y a 603 no constantes. Analizando

\footnotetext{
* Artigo extraído da dissertação de ALBUQUERQUE, C.C. Termos da linguagem especial de Enfermagem identificados em registros de uma UTI Neonatal. 2006. 100 pág. Dissertação (Mestrado). Centro de Ciências da Saúde / Universidade Federal da Paraíba, João Pessoa. Pesquisa desenvolvida com auxílio bolsa CNPq.

1 Enfermeira. Mestranda do Programa de Pós-Graduação em Enfermagem do CCS/UFPB. João Pessoa/PB. E-mail: candi albuquerque@hotmail.com.

Enfermeira. Doutora em Enfermagem. Docente do Departamento de Enfermagem em Saúde Publica e Psiquiatria e do Programa de Pós-Graduação em Enfermagem, do Centro de Ciências da Saúde da UFPB. Pesquisador CNPq. Orientadora. João Pessoa/PB. E-mail: candi albuquerque@hotmail.com

3 Enfermeira. Doutora em Enfermagem. Docente Aposentada do Departamento de Enfermagem em Saúde Publica e Psiquiatria. Docente do Programa de Pós-Graduação em Enfermagem, do Centro de Ciências da Saúde da UFPB. Pesquisador CNPq. Orientadora. João Pessoa/PB. E-mail: tegarcia@ccs.ufpb.br
} 
los 166 términos constantes, 45 pertenecían al eje Foco, 8 al eje Juicio, 23 al eje Meios, 39 al eje Acción, 12 al eje Time, 30 al eje Localización, y 4 al eje Cliente, analizados segundo su relevancia para la UTIN. De acuerdo con los resultados obtenidos, puede concluirse que se alcanzaron los objetivos que orientaron esta investigación y el camino metodológico fue apropiado. Considerase el estudio

\section{INTRODUÇÃO}

A Enfermagem é uma disciplina relativamente nova, pois foi considerada ciência moderna em meados do século XIX, com Florence Nightingale, razão pela qual, apresenta, ainda, um corpo de conhecimentos em construção, cujo foco necessita de ser definido claramente. A necessidade de se instituir o cerne desse conhecimento exigiu a definição de seus conceitos próprios, seu foco de atenção e uma linguagem especial determinada, pois uma disciplina só é reconhecida como ciência, entre outros aspectos, pela determinação de sua conceitualização e fenômenos específicos.

Tornou-se vital para a evolução da Enfermagem como ciência, o desenvolvimento de seu corpo de conhecimentos, através do desenvolvimento de modelos conceituais e teorias, os quais selecionam e inter-relacionam conceitos, a partir de diferentes visões de mundo, refletindo a natureza e o cerne da profissão, fornecendo focos para a mesma e oferecendo roupas para um "esqueleto desnudo". (GORDON apud GARCIA \& NÓBREGA, 2004).

CARVALHO \& CASTRO (1979) afirmam que o desenvolvimento da Enfermagem, como profissão, através da história, só será possível mediante suas adaptações às novas expectativas e necessidades da sociedade, oriundas do desenvolvimento científico. As acomodações às exigências sociais caracterizam-se como uma desconstrução de um conhecimento para sua reconstrução, fato que seria denominado crise do conhecimento, segundo CAPRA (2001), que resultaria, aqui, numa redefinição dos domínios da Enfermagem.

Tendo iniciado sua caminhada para a adoção de uma prática baseada em conhecimentos científicos, a Enfermagem abandonou gradativamente sua postura de "atividade caritativa, intuitiva e empírica" (POSSARI, 2005, p.1), e vem tentando definir o foco de sua prática, o qual, apesar de algumas divergências, se aceita que está direcionado, fundamentalmente, no cuidar do paciente, que deve ser holístico, voltado para as necessidades do cliente, seja ele indivíduo, família ou comunidade.

Com esse intuito, os diversos conceitos, teorias e modelos específicos da Enfermagem que estão sendo desenvolvidos têm a finalidade de fundamentar a prática assistencial, para que as ações sejam planejadas, determinadas e gerenciadas para o importante para el conocimiento del vocabulario usado en UTIN y para la construcción de una nomenclatura específica para esta unidad.

PALABRAS CLAVE: Enfermería; Linguagem; Registros de Enfermería, Terminología.

cuidado, seja ele individual ou coletivo, a fim de que seu registro permita uma avaliação e a geração de conhecimentos.

Nessa perspectiva construtiva de conhecimentos e transformativa de uma prática acrítica, verifica-se um investimento nas pesquisas e um avanço acelerado na construção do conhecimento objetivando-se uma melhor condução na delimitação do papel da enfermagem. Assim, têm-se elegido esforços sobre a orientação da prática profissional, que orientem a uniformização das ações na prática profissional, e, assim, impulsione o desenvolvimento de uma prática científica, como também, a produção de uma linguagem específica utilizada na profissão, que a represente (VIRGÍNIO \& NÓBREGA, 2004).

Observa-se, na Enfermagem, um acúmulo de conhecimentos sem caráter científico, com execução de atividades baseadas em normas e rotinas repetidas, sem reflexão, muitas vezes subordinadas a outras profissões, tornando suas atividades mecânicas, afastando-se das especificidades inerentes à profissão, desempenhando-se em decorrência disso, ações sem nenhuma crítica e uma assistência de enfermagem descontínua e desqualificada (CAMPEDELLI et al., 1992).

Percebeu-se a necessidade de se desenvolver um método de trabalho que tornasse a prática da Enfermagem visível, mostrando o benefício das atividades desenvolvidas e proporcionando um cuidado de enfermagem com qualidade, através de sua crítica e avaliação contínua, o que poderia tornar a avaliação do cuidado eficaz, além de proporcionar a avaliação dos custos dessa prática.

Para MARIN et al. (2004), qualificar o cuidado de enfermagem é a melhor forma de se atender às condições do usuário, através dos cuidados a ele prestados, levando-se em conta seus objetivos, suas estratégias e os recursos disponíveis. Nessa perspectiva, um processo de enfermagem é aplicável, pois possibilita uma visão ampliada da assistência, melhora a interação enfermeiro/paciente, facilita a avaliação, possibilita o planejamento de recursos materiais e humanos.

O conceito de processo de enfermagem não é novo, uma vez que sua concepção já tinha sido empregada por Florence, no século XIX, sobre a necessidade de se fazer julgamento sobre situações observadas. Essa noção foi aprimorada ao longo dos anos, pela implementação de alguns métodos de 
estudo, como estudos de caso e através da tentativa para se desenvolver um modelo operacional para a prática. Em conseqüência desses fatores, chegou-se à denominação de processo de enfermagem, por Ida Jean Orlando no início da década de 1960 (GARCIA \& NÓBREGA, 2002). Era constituído de algumas etapas e tinha como objetivo principal 0 desenvolvimento de uma prática individual, na qual o enfermeiro pudesse detectar um problema ou necessidades nos pacientes, e, a partir daí, desenvolver um plano de cuidados específicos.

$\mathrm{Na}$ perspectiva de que o Processo de Enfermagem serve como guia prático para fornecer autonomia profissional, acredita-se que ele seja, além disso, uma maneira de a Enfermagem concretizar a proposta de promover, manter ou restaurar o nível de saúde do paciente, através do cuidado e da promoção do autocuidado, como também documentar sua prática profissional, visando à avaliação da qualidade da assistência prestada. Assim, o processo de enfermagem pode contribuir para delimitar o campo prático e teórico, fornecendo normas diretivas para a organização do conhecimento da profissão, fundamentando uma prática autônoma, pois provê o domínio profissional de características únicas que possibilitam uma exposição à observação e à crítica dos seus pares, dos outros profissionais da equipe e, acima de tudo, da população que faz uso dos serviços de saúde (CRUZ, 1997). A mesma autora cita que, através dos seus componentes, o processo de enfermagem torna-se indicador das necessidades dos cuidados de enfermagem da pessoa ou do grupo que está sendo cuidado. Essas necessidades de cuidados de enfermagem compõem uma constelação de focos aos quais se devem dirigir o desenvolvimento do corpo de conhecimentos, porque, uma vez identificados os fenômenos clínicos de interesse para a Enfermagem, haverá sempre um ponto de partida para as pesquisas.

Isso faz com que se remeta a diversos problemas que podem surgir da inexistência ou falha na implementação do método, como também, do registro incompleto, ou sua falta, como complementação do processo. Alguns desses problemas seriam a falta de visibilidade da Enfermagem, a impossibilidade de avaliação da sua prática, e, talvez, em primeiro grau de relevância, a impossibilidade de avaliação do cuidado prestado ao paciente, levando-o a uma prática pouco eficaz.

A avaliação do registro da assistência aos pacientes sempre foi uma parte essencial do desenvolvimento do cuidado de enfermagem. Apesar disso, ainda não é possível descrever o conteúdo da Enfermagem prática, pois a linguagem utilizada no cuidado não se tornou suficientemente unívoca para satisfazer as exigências edificadoras de sistemas de dados (KARKKAINEN \& ERIKSSON, 2003).

Enfatizando essa problemática, NÓBREGA \& GARCIA (2005), abordam que a falta de uma linguagem universal que estabeleça e defina a prática profissional tem comprometido a evolução da enfermagem como ciência. Essa problemática levou ao estabelecimento de algumas alternativas, como a produção de modelos conceituais, o desenvolvimento de pesquisas relacionadas com o estudo de conceitos e a introdução do processo de enfermagem, o qual se pode entender como o modelo metodológico de que se lança mão, tanto para tomar decisões acerca do cuidado, quanto para organizar as condições necessárias para que o mesmo seja realizado.

A documentação é exigida para substanciar a responsabilidade sobre processos e resultados do cuidado. Os dados oriundos são requisitos para a condução de pesquisas empíricas, assim como uma necessidade para investigadores responderem a perguntas de pesquisas relacionadas com a assistência, a fim de identificar melhores práticas, desenvolver e testar modelos de cuidado, desenhar modelos de suporte de decisão e determinar uma utilização eficiente e efetiva de recursos (McCORMICK \& JONES, 1998).

Acredita-se que a percepção da relevância do desenvolvimento de estudos sobre a linguagem utilizada pelos enfermeiros para exprimir sua prática deu-se pela implementação do processo de enfermagem, pois o mesmo facilitou e organizou a realização do cuidado, assim como a análise de seu registro, que por sua vez, impulsionou o desenvolvimento de sistemas relacionados com cada etapa do processo, com a identificação do vocabulário utilizado para diagnósticos, intervenções e resultados.

Nessa perspectiva, diversas terminologias padronizadas têm sido desenvolvidas, proporcionando riqueza na possibilidade de representação dos fenômenos de enfermagem e domínios da prática, como também, atendendo às necessidades de documentação e análises da Enfermagem, de acordo com as fases do Processo de Enfermagem (MARIN, 2001).

Esse trabalho terminológico consiste, então, em representar um campo conceptual e estabelecer denominações precisas que garantam uma comunicação intersubjetiva, que deve ser feita em utilização de sistemas de classificação dos termos utilizados na disciplina, para emprego dentro da Sistematização da Assistência de Enfermagem (SAE) e unificação do vocabulário (BITTENCOURT et al., 2002).

Verifica-se, na revisão da literatura da área, que a diversidade de sistemas de classificação desenvolvidos no mundo, direcionados para as realidades e exigências locais, estão voltados, muitas vezes, para uma única etapa do processo de enfermagem. Contudo, percebeu-se que essa gama de classificações não fornecia meios práticos, nem para agregar, nem para comparar os dados (MARIN, 2001), o que levou o International Council of Nurses (ICN) a construir uma Classificação Internacional para 
a Prática da Enfermagem, que se tornaria um Marco Unificador.

Baseando-se nisso, percebeu-se a relevância da identificação de uma linguagem específica utilizada pelos enfermeiros, ou seja, a identificação de todo o vocabulário utilizado, na prática, pelos mesmos, principalmente para o registro de suas atividades desenvolvidas.

O motivo do aparente desinteresse de alguns profissionais pelo aprimoramento da prática tem sido foco de questionamento, pois subestimando o registro da assistência e sua avaliação, como fase do processo de enfermagem e descrevendo suas intervenções de forma acrítica, muitas vezes realizando cópias, ou deixando de determiná-las o enfermeiro não dá visibilidade ao seu trabalho.

Com 0 ingresso no Programa de PósGraduação em Enfermagem, nível Mestrado, e, a partir de estudos sobre o Processo de Enfermagem e os Sistemas de Classificação, tomou-se conhecimento do projeto de Sistematização da Assistência da Enfermagem desenvolvido no Hospital Universitário Lauro Wanderley da Universidade Federal da Paraíba (HULW/UFPB) e dos projetos de pesquisas que, tinha como um dos objetivos gerais, mapear os termos atribuídos a fenômenos/diagnósticos/problemas e a ações/intervenções/prescrições na prática de enfermagem desse hospital (NÓBREGA \& GARCIA, 2000). Esses projetos de pesquisa já tinham feito o mapeamento dos termos utilizados em registros profissionais pelos componentes da equipe de enfermagem de todas as unidades clínicas da instituição, com exceção da Unidade de Terapia Intensiva Neonatal (UTIN).

Esse fato gerou motivação para o investimento no presente estudo para o desenvolvimento de uma terminologia específica para a assistência de enfermagem na UTIN, com o intuito de desenvolver conhecimentos específicos e novos, que serão úteis e relevantes para a futura implementação do processo de enfermagem, melhorando a qualidade da assistência de enfermagem prestada aos pacientes de risco.

Para tanto se questiona: que termos da linguagem especial de enfermagem são utilizados pelos componentes da equipe de enfermagem da UTIN do HULW/UFPB, em seus registros da assistência ao recém-nascido? Esses termos estão incluídos na Classificação Internacional para a Prática da Enfermagem - CIPE ${ }^{\circledR}$ Versão 1?

Partindo dessas questões norteadoras o presente estudo tem como objetivos: Identificar os termos empregados pelos componentes da equipe de enfermagem, nos registros em prontuários da UTIN do HULW/UFPB; fazer o mapeamento cruzado dos termos identificados com os constantes na CIPE $^{\circledR}$ Versão 1, de modo a verificar quais os incluídos e os não incluídos e categorizar os termos identificados como não constantes, segundo os eixos da $\mathrm{CIPE}^{\circledR}$
Versão 1.

\section{PERCURSO METODOLÓGICO}

Trata-se de um estudo com abordagem descritiva, em que se utilizou o método retrospectivo, para a coleta de dados em registros de enfermagem e o processo de mapeamento para análise dos dados contidos nesses registros. A análise foi realizada pelo método de cross-mapping, que consiste em mapear os termos em questão, e compará-los com os termos de um modelo de referência, a CIPE ${ }^{\circledR}$ Versão 1 , de modo a se identificar os que já fazem parte e os que ainda não estão inseridos neste modelo de referência.

A pesquisa foi desenvolvida na UTI Neonatal do Hospital Universitário Lauro Wanderley - HULW, da Universidade Federal da Paraíba - UFPB, após a aprovação do projeto pelo Comitê de Ética em Pesquisa (protocolo 010/06) e a autorização da direção do HULW/UFPB, para o manuseio e coleta de dados nos prontuários dos pacientes. A aprovação pelo Comitê de Ética e a autorização da direção da instituição para manuseio e coleta de dados nos prontuários dos pacientes representam a garantia da observância dos aspectos éticos preconizados na Resolução N¹96/96, do Ministério da Saúde (BRASIL, 1996).

Para o estudo, foram utilizados todos os prontuários dos pacientes admitidos no serviço, desde a inauguração da unidade até a data da coleta. Como critério de inclusão, levou-se em consideração os prontuários que continham registros feitos pelos componentes da equipe de enfermagem que pudessem levar à identificação dos termos da linguagem especial de enfermagem. Desse conjunto, que constava de um registro de admissão de 43 prontuários, só se obteve acesso a 13 deles, pois muitos desses prontuários não foram encontrados no Serviço de Arquivo Médico do hospital.

Foram considerados como população do estudo os prontuários de todos os pacientes admitidos no serviço, desde sua inauguração, em $11 / 11 / 2004$, até a data da coleta, perfazendo um total de 43 prontuários. Como critério de inclusão, levou-se em consideração os prontuários que continham registros feitos pelos componentes da equipe de enfermagem que pudessem levar à identificação dos termos da linguagem especial de enfermagem. Dos 43 prontuários só se obteve acesso a 13 deles, pois muitos dos mesmos não foram encontrados no Serviço de Arquivo Médico (SAME) do hospital.

Para a coleta de dados nos prontuários, foi utilizado um instrumento, contendo espaço para a transcrição literal de todos os registros feitos pelos componentes da equipe de enfermagem. A partir dessa transcrição, todos os conteúdos dos prontuários foram digitados, para formar o corpus de análise. Esses passaram por um processo de análise, para se identificar a relevância de seu conteúdo, e, então, realizar a extração de termos. 
Em um primeiro momento, iniciou-se a decomposição do texto em termos, o que gerou uma listagem imensa de termos simples, que, na medida em que se avançava na decomposição, tornava-se mais difícil identificá-los, pois não havia referência contextual para reconhecê-los.

Diante dessa problemática, e após estudos sobre Terminologia, modificou-se o método de trabalho e passou-se a trabalhar com arquivos em que se colocava uma tabela com o texto fragmentado em parágrafos, para facilitar a análise, e, em outra coluna, os termos extraídos.

Nessa etapa, seguiram-se as orientações metodológicas do trabalho terminológico apresentado por PAVEL \& NOLET (2003), que ressalta a importância do contexto em que os termos se apresentam assim como também, amplia as possibilidades de inclusão de termos compostos, sintagmas, fraseologias. Dessa forma, iniciou-se a extração de termos notórios e fraseologias, das quais foram extraídos os termos simples.

Em uma segunda etapa, realizou-se a normalização dos termos extraídos, dos quais foram quantificadas as repetições, corrigida a ortografia, analisada a sinonímia e realizadas algumas adequações de termos referidos pela $\mathrm{CIPE}^{\circledR} 1$, com posterior inserção em uma planilha do Excel for Windows, chegando a uma totalização de 869 termos.

Esse banco de dados foi submetido ao processo de mapeamento cruzado, que faz a ligação desses termos aos conceitos apresentados nos eixos da CIPE $^{\circledR}$ Versão 1 , com a identificação dos termos constantes e não constantes nessa Classificação. Para isso, foi utilizado o programa Access for Windows para importar, da planilha do Excel, o banco de dados, com a finalidade de se construir uma tabela com os termos da linguagem especial de enfermagem identificados no estudo, classificados segundo os sete eixos constantes na CIPE ${ }^{\circledR}$ Versão 1.

\section{RESULTADOS E DISCUSSÃO}

Atendendo aos objetivos propostos e critérios metodológicos adotados neste estudo, realizou-se a coleta de dados, em um primeiro momento, na UTIN, utilizando o livro de registro de admissões para obter os números de registros dos pacientes internos até a data da coleta. Foram identificados 43 registros de admissões a partir da data da primeira admissão, quando a unidade foi inaugurada, até o dia 06.03.06. Através desses números de registro, localizou-se, no Serviço de Arquivo Médico - SAME, alguns prontuários que continham registro de enfermagem adequado para a coleta de dados, levando-se em consideração que essa foi uma condição para inclusão do prontuário no estudo. Nessa "busca" realizada no SAME, observou-se inúmeras falhas nesse método de armazenamento de dados escritos, especialmente pela falta de muitos dos prontuários procurados, pois dos 43 registros só foram localizados 13 prontuários, que, com exceção daqueles que estavam sendo utilizados por nova internação do paciente, havia prontuários abertos, sem nenhum registro realizado, mais de um prontuário para o mesmo paciente, erros que possivelmente seriam resolvidos com a utilização de um registro eletrônico.

Ao analisar os dados de identificação coletados nos prontuários, verificou-se que a idade das crianças internadas variou entre 1 e 68 dias de vida, $60 \%$ eram do sexo masculino e $40 \%$ do sexo feminino, havendo, ainda, uma média de 14 dias de internação. Esses dados apontam um perfil do paciente neonatal crítico que necessita de cuidados intensivos, o qual é admitido, na maioria das vezes, após o nascimento, por problemas de alto risco para a vida, como refere KENNER (2001). Cabe salientar que todos os registros textuais de enfermagem encontrados foram realizados por enfermeiras, em registros de evolução do paciente, não sendo identificada nenhuma fase do processo de enfermagem aplicada. Em contrapartida, percebe-se que existe um opulente registro de informações mostrando que há conscientização ética das enfermeiras sobre a importância desse papel. Constatou-se que, nessa unidade, as técnicas de enfermagem registram apenas em um instrumento de boletim diário seus cuidados de rotina.

Seguindo as etapas da metodologia proposta para analisar esses registros, chegou-se à extração inicial dos 2.047 termos, os quais passaram por uma primeira normalização, chegando-se a 869 termos, levando em consideração que foram excluídos o "lixo terminológico", conjugações verbais, repetições, descritas as siglas e acrônimos, uniformizado gênero e número, corrigido a ortografia, além da contabilização do número de ocorrências dos termos. Os 869 termos passaram por uma nova normalização para exclusão de verbos que não eram ação de enfermagem, inclusão de sintagmas que haviam sido dissociados, chegando com isso a 764 termos. A partir dos 764 termos, que constitui o banco de dados de termos da UTIN, foi feito o mapeamento cruzado com os termos da CIPE ${ }^{\circledR}$ Versão 1, evidenciando que 161 são termos constantes e 603 termos não constantes nessa Classificação.

O resultado revela que os componentes da equipe de enfermagem da referida UTIN, utilizam, com maior freqüência, os termos que não constam na $\mathrm{CIPE}^{\circledR} 1$, apresentando uma linguagem específica e peculiar, que é comum entre eles, e que se mostra expressiva mesmo sem a implementação de nenhum sistema de classificação, nem mesmo da SAE, demonstrando o quão são necessários os estudos para a normalização desse vocabulário tão expressivo.

Esse resultado não difere muito dos estudos anteriores, realizados na mesma Instituição, como o de NÓBREGA et al. (2003), que mostra que a linguagem utilizada pela Enfermagem não está uniformizada e que os profissionais não utilizam, na 
prática, nenhum tipo de classificação que possa ser identificado. Nesse sentido, pode-se inferir que as dificuldades de implantação do processo de enfermagem referidas em algumas pesquisas também podem assemelhar-se às dificuldades para se uniformizar a linguagem utilizada para o registro desse processo.
Analisados os 161 termos constantes na CIPE $^{\circledR}$ 1 , observou-se que 45 termos pertencem ao eixo Foco, 8 são do eixo Julgamento, 23 do eixo Meios, 39 do eixo Ação, 12 do eixo Tempo, 30 do eixo Localização e 4 do eixo Cliente, conforme pode ser visualizado no Gráfico 1.

Gráfico 1 - Termos identificados nos registros de componentes da equipe de enfermagem em prontuários da Unidade de Terapia Intensiva Neonatal e classificados segundo o Modelo dos 7 Eixos da CIPE ${ }^{\circledR}$ 1. João Pessoa, 2006.

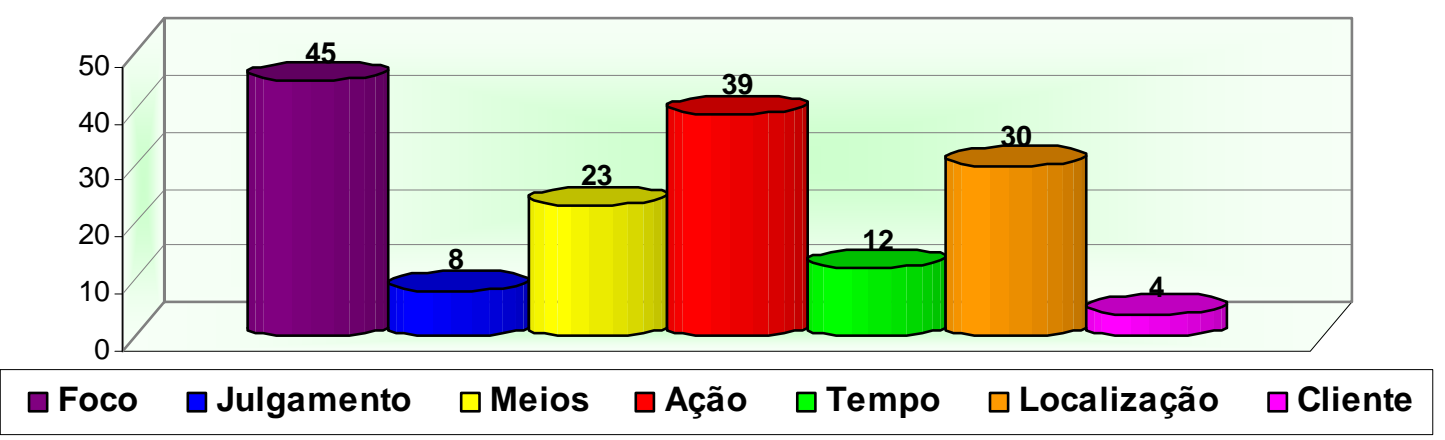

Visualiza-se no Gráfico 1, uma prevalência de termos constantes nos eixos Foco, Ação e Localização, em particular no eixo Foco. Esse aspecto pode ser explicado, pois o mesmo direciona o foco de atenção da assistência ao paciente. Esse resultado também está relacionado ao perfil dos pacientes que foram referidos nos registros analisados, já que os mesmos recebiam cuidados intensivos e eram neonatos. Assim, acredita-se que a focalização da assistência esteve voltada, principalmente, para os fenômenos apresentados pelos mesmos, tanto para o julgamento de uma determinada situação de risco específica, como em relação à ação produzida sobre a mesma. Deduz-se, então, que a localização esteve registrada quando referida para o mesmo fenômeno, no intuito de propiciar um cuidado mais preciso.

A análise dos termos identificados como constantes foi realizada, segundo a árvore temática proposta para o estudo, pois é preciso delimitá-los, dentro de suas áreas temáticas pertinentes, para se visualizar as relações hierárquicas dos conceitos em estudo. PAVEL \& NOLET (2002) referem que os conhecimentos de uma área se estruturam em uma terminologia, conforme as relações hierárquicas e lógicas entre os conceitos que a integram.

Analisando os 45 termos extraídos dos registros, e classificados como pertencem ao eixo FOCO, evidencia-se que 7 estão no conceito ENTIDADE, 7 no conceito STATUS e 31 no conceito PROCESSO. Entidade é definido pelo CIE (2006, p. $35)$, como sendo "[...] algo que existe como uma unidade particular e abstrata; existência de algo para além das suas propriedades." Neste conceito foram classificados 7 termos, sendo 3 no subconceito Substância corporal: sangue, fezes, amostra; 3 no subconceito Sistema Corporal: sistema cardiovascular, sistema gastrointestinal, sistema respiratório; 1 no subconceito Resultado; 1 no subconceito Taxa: freqüência cardíaca. No conceito STATUS definido pelo CIE (2006, p. 91), como "[...] condição da pessoa relativamente a outras, posição relativa de uma pessoa.", foram identificados 6 termos, sendo 2 no subconceito Acesso: acesso, acesso intravenoso; 3 no subconceito Estado Fisiológico: comprimento, peso, temperatura; apenas 1 termo no subconceito Ritmo: ritmo cardíaco. Quanto ao conceito PROCESSO, definido pelo CIE (2006, p. 39), como sendo "[...] conjunto de funções ou ações para atingir um resultado.", foram identificados 31 termos, sendo 25 no subconceito Processo Corporal: aspiração, bradicardia, constipação, desidratação, dispnéia, edema, eliminação, ferida, gravidez, hematoma, hipotermia, hipóxia, movimento, pele seca, reflexo, reflexo de sucção, regurgitação, sangramento, sinal, sono, tosse, tremor, ventilação, vômito; e 6 termos no subconceito Comportamento: amamentação exclusiva ao seio, nutrição, padrão respiratório, reatividade, rotina, sucção.

Observa-se na distribuição dos termos nesses três blocos de conceitos, uma predominância no conceito PROCESSO, no subconceito Processo Corporal, levando-nos a considerar que esse resultado mostra uma característica da população do estudo, pois nesse conceito encontramos termos relacionados a processos vitais, representando uma necessidade peculiar da assistência ao RN voltada para manutenção da vida.

Os termos amamentação exclusiva ao seio, reflexo de sucção e sucção, constantes na CIPE $^{\circledR}$ Versão 1 e identificados no estudo são de grande relevância para a linguagem da especialidade da qual 
coletamos os dados, visto que os registros estão voltados, principalmente, para recém-nascidos e que termos como estes retratam bem as especificidades deste tipo de paciente.

Analisando a árvore conceitual do eixo Julgamento e a distribuição dos termos constantes na $\mathrm{CIPE}^{\circledR}$ Versão 1, evidencia-se que esse eixo apresenta dois blocos conceituais, contemplados com os 9 termos constantes nesse eixo. No conceito JULGAMENTO POSITIVO OU NEGATIVO, definido pelo CIE (2006, p. 107), como "[...] julgar positiva ou negativamente.", identificamos apenas 1 termo no subconceito Prejudicado. No conceito ESTADO, definido pelo CIE (2006, p. 107), como sendo "Julgamento", encontramos a maior parte dos termos constantes nesse eixo, com 2 termos classificados em cada subconceito a seguir: Nível absoluto: elevado, baixo; Dimensão: parcial, total; Estado de normalidade: anormal, normal; Tamanho: grande e pequeno.

Os termos identificados como constantes neste eixo estão predominantemente distribuídos no bloco conceitual Estado, assim como os termos do eixo Foco estiveram concentrados principalmente no bloco Processo. Relacionando estes resultados, visto que o julgamento é feito sobre um foco de atenção, inferimos que os julgamentos realizados focalizaram os processos, em particular os corporais, ressaltando que os registros da assistência estão voltados, principalmente, para o cuidado das necessidades biológicas, com o intuito de ajudar na manutenção da vida, adaptação ao meio ambiente e no auxílio aos processos fisiológicos.

A análise dessa árvore conceitual nos fez perceber a falta de julgamentos de risco, potencial e atual para os focos de atenção. Não se encontrou nos registros nenhum julgamento sobre a potencialidade do problema, ocorrência importante, pois com isso ressalta-se que não existe uma preocupação sobre a prevenção, por exemplo. A assistência parece estar voltada para os problemas presentes no momento, estando o julgamento atual subentendido.

A árvore conceitual do eixo MEIOS mostra uma distribuição homogênea dos 23 termos entre quase todos os blocos conceituais, demonstrando a diversidade de recursos tecnológicos e terapêuticos empregados na assistência. Nessa árvore, encontram-se seis blocos de conceitos mais abrangentes: ARTEFATO, PRESTADOR DE CUIDADO, SERVIÇO DE SAÚDE, MATERIAL, TÉCNICA, TERAPIA, todos definidos pelo CIE (2006, p.126) como sendo "Meios". Esses blocos, diferentemente do que aconteceu nos outros eixos não apresentam subconceitos. No conceito ARTEFATO, foram identificados 12 termos: cateter, compressa, dreno, fralda, incubadora, leito, máscara, plano, respirador, sonda, sutura, tubo endotraqueal. O conceito PRESTADOR DE CUIDADO englobou 3 termos constantes: cirurgião, enfermeira, médico; MATERIAL, com 4 termos: antibiótico, droga, material, solução; TÉCNICA, com 2 termos: cesariana, cirurgia; TERAPIA com 2 termos: hemoterapia e oxigenoterapia. A maioria dos conceitos foi contemplada, à exceção dos serviços de saúde. Nesse conceito consideraram-se como sinônimos àqueles que mantiveram relação conceitual com termos presentes na CIPE, são eles: equipe de enfermagem e fisioterapia (termos extraídos) e serviço de enfermagem e serviço de fisioterapia (termos CIPE), respectivamente. Esse resultado evidencia que nesse eixo existe uma predominância de termos relacionados aos conceitos de artefato e material, o que revela uma característica do registro realizado na UTIN. Parece haver uma preocupação em relatar os materiais utilizados nos procedimentos e/ou terapias e aqueles que servem para monitorização do RN. Sendo estes dispositivos utilizados para colaborar com as ações sobre os processos corporais, evidencia-se, mais uma vez, que há uma inclinação para o modelo biologicista.

$\mathrm{Na}$ árvore conceitual do eixo AÇÃO, os 38 termos constantes foram distribuiu em quatro dos cinco blocos conceituais apresentados na $\mathrm{CIPE}^{\circledR}$ Versão 1: ATENDER, DETERMINAR, INFORMAR, GERENCIAR e DESEMPENHAR. O conceito INFORMAR é definido na CIPE $^{\circledR}$ Versão 1 como sendo "[...] comunicar alguma coisa a alguém" (CIE, 2006 , p. 136), foi representado por 1 termo constante - Registrar -, o que leva a concluir que as atividades neste âmbito relacionados aos termos instruir, ensinar, explicar, orientar, enfim, ações voltadas para a família em se tratando de pacientes neonatais, não foram contempladas. $O$ fato desses termos não estarem sendo incluídos nos registros de enfermagem, não permite inferir que essas atividades não estejam sendo realizadas. Entretanto, chama a atenção para a importância de a Enfermagem contemplar esses aspectos nos registros da sua prática assistencial a fim de que a visibilidade da assistência ao RN e sua família possa ser identificada na forma de documentação do cuidado. O único termo identificado como constante neste conceito foi registrar, evidenciando literalmente que há uma preocupação sobre a atividade do registro. É inegável que o registro feito na unidade é ostensivo com relação à quantidade, mas levanta-se alguns questionamentos quanto ao seu conteúdo, que claramente não está adequado ao processo de enfermagem, mas sim ao enfoque no modelo biomédico; e quanto a lacuna com relação à assistência voltada para a família, seja esta como participante do cuidado ou como foco de atenção dos cuidados da Enfermagem.

No conceito ATENDER, definido pelo CIE (2006, p. 131), como sendo "[...] estar atento a, de serviço a, ou a tomar conta de alguém ou alguma coisa.", encontramos 6 termos nos seguintes subconceitos: Assistir: promover; Prevenir: evitar, prevenir, proteger; Tratar: evoluir; Relacionar: contatar. No conceito DETERMINAR, definido na 
$\mathrm{CIPE}^{\circledR}$ Versão 1 como sendo "[...] descobrir ou estabelecer com precisão a presença de alguma coisa." (CIE, 2006, p.134), foram encontrados 5 termos, 4 no subconceito Avaliar: avaliar, medir, pesar, verificar; e 1 termo no subconceito Monitorizar. O conceito GERENCIAR, definido como sendo "[...] estar encarregado de, e organizar para alguém ou alguma coisa." (CIE, 2006, p. 137), englobou 13 termos que ficaram distribuídos nos subconceitos, Distribuir com 2 termos: administrar, oferecer; Ordenar com 1 termo: prescrever; Organizar com 10 termos: adaptar, aumentar, desmamar, diminuir, estabilizar, imobilizar, iniciar, manter, regular, referir. Quanto ao conceito DESEMPENHAR, definido na CIPE ${ }^{\circledR}$ Versão 1 como sendo "[...] executar uma tarefa técnica." (CIE, 2006, p. 141), encontramos 14 subconceitos contendo termos constantes, o subconceito Limpar com 2 termos: aspirar, drenar; Posicionar com 2 termos: posicionar, transferir; Inserir com 2 termos: intubar, puncionar; e os subconceitos que se apresentaram como termos constantes: Trocar, Cobrir, Suturar, Instalar, Manipular, Alimentar, Demonstrar, Transportar.

No eixo Ação verifica-se uma distribuição da maioria dos termos entre os conceitos desempenhar e gerenciar, relacionados ao desempenho de técnicas para com o paciente ou com o manuseio de materiais, e com o gerenciamento de ações para o paciente ou o gerenciamento de situações, respectivamente. Este resultado mostra que as ações que estão sendo realizadas são voltadas para as técnicas, principalmente, e são registrados os aspectos mais subjetivos da assistência de enfermagem.

Ao analisar a árvore conceitual do eixo TEMPO, observa-se que os 12 termos constantes estão distribuídos em três dos 6 blocos conceituais apresentados na CIPE $^{\circledR}$ Versão 1: DURAÇÃO, FREQÜÊNCIA, INÍCIO, SITUAÇÃO, PONTO NO TEMPO e SEQÜÊNCIA TEMPORAL. O termo INÍCIO, definido pelo CIE (2006, p. 149) como sendo "Tempo", foi identificado como o nome do próprio bloco conceitual. No conceito SITUAÇÃO, também definido pelo CIE (2006, p. 140) como sendo "Tempo", encontramos 3 termos no subconceito Evento: admissão, nascimento e parto. No conceito INTERVALO DE TEMPO, também definido pelo CIE (2006, p. 140) como sendo "Tempo", evidenciamos 7 termos que são subconceitos: amanhã, dia, hoje, manhã, noite, presente, tarde. No conceito SEQÜẾNCIA TEMPORAL, definido na $\mathrm{CIPE}^{\circledR}$ Versão 1 como sendo "[...] seqüência de uma coisa atrás de outra, uma ordem de sucessão." (CIE, 2006, p. 152), encontramos apenas um termo que também é subconceito: contínuo. Neste eixo encontra-se a maioria dos termos relacionados ao período do tempo em que uma ação foi realizada; ao planejamento de uma ação; à delimitação no tempo de quando um sinal/sintoma apareceu no RN, entre outros. Percebese, também, que houve um subregistro dos termos do conceito freqüência e início, mas cabe ressaltar que ocorreram registros sobre o número de vezes de uma ocorrência através da utilização de sintagmas, como 3 vezes ao dia e a cada 24 horas.

A árvore conceitual do eixo LOCALIZAÇÃO, evidencia a distribuição dos 30 termos constantes em dois blocos conceituais dos três existentes na CIPE $^{\circledR}$ : POSIÇÃO e ESTRUTURA, definidos pelo CIE (2006, p. 155-167), como sendo "Localização". No conceito POSIÇÃO, identificou-se 9 termos, incluindo o próprio termo do bloco conceitual, e outros nos subconceitos: anterior, central, direito,esquerda, inferior, periférico, posterior, superior. Os outros termos estão distribuídos no conceito ESTRUTURA, que apresenta o subconceito Estrutura do Corpo com 16 termos constantes: abdome, cabeça, cavidade oral, couro cabeludo, olho, pele, região axilar, região umbilical, tórax, veia, via aérea, via gastrointestinal, via intramuscular, via nasal, via oral, via parenteral; 5 termos no subconceito Estrutura Social: ambulatório, berçário, clínica, clínica obstétrica, hospital. Percebese que nesse eixo houve uma predominância sobre o conceito estrutura do corpo, confirmando o que já foi identificado nos outros eixos, ou seja, existe um enfoque sobre o biológico do paciente, caracterizando mais objetivamente seus processos patológicos. Ressaltamos que as notificações foram realizadas com relação apenas ao $\mathrm{RN}$, e não houve nenhum registro relacionado à mãe.

$\mathrm{Na}$ árvore conceitual do eixo Cliente os 4 termos constantes foram identificados em dois blocos conceituais: GRUPO e INDIVÍDUO. No conceito GRUPO, definido na CIPE $^{\circledR}$ Versão 1 como sendo "[...] um agrupamento de seres de humanos vistos como uma unidade social ou como um todo coletivo [...]" (CIE, 2006, p. 171), foi identificado 1 termo que é subconceito: família, e no conceito INDIVÍDUO, definido como sendo "[...] um ser humano." (CIE, 2006, p. 171), foram encontrados três termos que também são subconceitos: lactente, paciente $e$ recém-nascido. Dentre tantos subconceitos existentes na $C I P E^{\circledR}$ Versão 1 para esse eixo, percebemos que os termos constantes indicam que a população do estudo é bastante específica, centrando-se no recémnascido.

Partindo dos termos mais freqüentes em cada eixo (Quadro 1), realizou-se uma análise da relevância de incidência dos mesmos, dentro do ambiente de terapia intensiva da neonatologia, ressaltando o motivo de sua freqüência nos registros de enfermagem dessa unidade de cuidado tão peculiar da pediatria. 
Quadro 1 - Distribuição por eixos dos termos mais freqüentes identificados nos registros de componentes da equipe de enfermagem da Unidade de Terapia Intensiva Neonatal e classificados como constantes segundo o Modelo dos 7 Eixos da CIPE $^{\circledR}$ 1. João Pessoa, 2006.

\begin{tabular}{|c|l|c|}
\hline Eixo & \multicolumn{1}{|c|}{ Termo } & Freqüência \\
\hline \multirow{2}{*}{ Foco } & Acesso & 138 \\
\cline { 2 - 3 } & Freqüência cardíaca & 132 \\
\cline { 2 - 3 } & Nutrição & 89 \\
\cline { 2 - 3 } & Sangue & 42 \\
\cline { 2 - 3 } & Peso & 36 \\
\cline { 2 - 3 } & Fezes & 33 \\
\hline \multirow{2}{*}{ Julgamento } & Elevado & 78 \\
$\mathrm{~N}=2$ & Total & 14 \\
\hline \multirow{2}{*}{$\mathrm{Neios}$} & Incubadora & 210 \\
\cline { 2 - 3 } & Médico & 21 \\
\cline { 2 - 3 } & Cirurgia & 12 \\
\hline \multirow{2}{*}{$\mathrm{Ação}$} & Evoluir & 186 \\
\cline { 2 - 3 } & Manter & 107 \\
\cline { 2 - 3 } & Aspirar & 74 \\
\hline Tempo & Presente & 330 \\
\cline { 2 - 3 } $\mathrm{N}=2$ & Parto & 12 \\
\hline Localização & Abdome & 336 \\
\cline { 2 - 3 } $\mathrm{N}=4$ & Periférico & 148 \\
\cline { 2 - 3 } & Pele & 87 \\
\cline { 2 - 3 } & Superior & 71 \\
\hline Cliente & Recém-nascido & 278 \\
$\mathrm{~N}=1$ & & \\
\hline
\end{tabular}

No eixo FOCO o termo Acesso foi o que apresentou maior freqüência, no contexto de acesso à via endovenosa do paciente, já que recém-nascidos internos em unidades de cuidados intensivos requerem a infusão de substâncias por acesso venoso por períodos muitas vezes prolongados (TOMA, 2004). Esse termo esteve, então, presente, de forma significante, indicando o tipo de acesso venoso implantado no paciente, suas condições e os procedimentos realizados sobre o mesmo.

Outro termo bastante encontrado nos registros foi Freqüência cardíaca, o segundo mais freqüente, em decorrência do alto nível de monitorização na UTIN. Entende-se sua alta incidência, pois a freqüência cardíaca é o principal determinante na decisão da reanimação (GUINSBURG \& ALMEIDA, 2006), manobra constantemente necessária para recém-nascidos.

Nutrição foi um termo utilizado referindo-se à necessidade de suporte nutricional, visto que há, em grande parcela dos pacientes internos em unidades de terapia intensiva, uma capacidade de digerir, absorver e metabolizar os nutrientes, de forma limitada, como também, uma capacidade gástrica limítrofe, muitas vezes, dependendo do grau de maturidade, com limitação no reflexo de sucção e deglutição. (HOCKENBERRY, 2006) Assim, essa atenção básica se fez constante nos registros da
UTIN, indicando sempre o tipo de nutrição, a quantidade e o tipo de dieta oferecida ao neonato, assim como, registros sobre sua resposta.

O termo Sangue apareceu em decorrência da alta taxa de realização de exames que utilizam esse material para análise, como a gasometria, realizada, no mínimo, uma vez por dia, em pacientes com quadro respiratório alterado; a glicosimetria, realizada diariamente para controle glicêmico desses pacientes que facilmente apresentam-se hipoglicêmicos, pois apresentam um metabolismo acelerado.

O Peso apresentou-se como um termo altamente significante, encontrado também na sua forma verbal pesar, indicando a necessidade dessa ação, para a verificação da evolução de massa corporal que está atrelada à maturação do recémnato e a sua adaptação ao meio extra-uterino. Essa relevância também é ressaltada pela sua inferência sobre a avaliação da idade gestacional e maturidade fetal, como também, pela sua relação com os altos índices de morbi-mortalidade nessa faixa etária. (HOCKENBERRY, 2006).

Fezes foi um termo bastante referenciado com sua coloração, aspecto e viscosidade. Sua relevância cabe dentro dos altos índices de doenças do trato gastrintestinal como as doenças congênitas, a exemplo da onfalocele e as infecções intestinais, entre os neonatos, o que faz a assistência da 
enfermagem estar mais voltada para a observância dos sinais e sintomas relacionados com o mesmo. Sua incidência esteve voltada para a observância, pelas enfermeiras, sobre as alterações dos padrões de evacuação que ocorre no período de adaptação do recém-nascido, o qual apresenta três fazes de eliminação intestinal: mecônio, fezes de transição e fezes lácteas. Assim, nota-se que houve um preocupação assistencial com as características fecais, para identificação de um funcionamento correto do trato gastrintestinal (HOCKENBERRY, 2006).

Dentro do eixo JULGAMENTO, encontrou-se o termo Elevado, em consonância com a forma mais utilizada para alteração de decúbito entre os recémnascidos, pois este decúbito facilita a respiração pela diminuição da pressão intraperitonial, como também evita a regurgitação que é tão freqüente nesses pacientes que ainda têm os mecanismos valvulares ineficazes (HOCKENBERRY, 2006).

Total fez-se presente no julgamento dos níveis de volume de substâncias administradas, dieta oferecida, como em referência a julgamento de componentes do sangue presentes em exames como o hemograma, dos quais as enfermeiras fazem relato nos seus registros para destaque sobre as outras informações.

No eixo MEIOS, o termo Incubadora foi identificado como o mais freqüente; este é um artefato ideal para acomodar um recém-nascido, no período neonatal precoce. Sua elevada incidência se deve a duas peculiaridades da população atendida pela UTIN: em primeiro lugar pela dificuldade natural em regular a temperatura dos recém-nascidos e segundo pela necessidade de acomodação em ambiente monitorado pelo provável risco de vida que apresentam. A incubadora é utilizada, preferencialmente, quando os recém-nascidos são pequenos para idade gestacional (PIG) e/ou de baixo peso (BP), pois a mesma mantém a temperatura sempre constante e filtra o ar, protegendo o recémnato do meio ambiente, enquanto ele não tem condições adaptativas satisfatórias. (MORAIS; CAMPOS \& SILVERSTRINI, 2005).

O termo Cirurgia surgiu nos registros relacionando-se às cirurgias eletivas para malformação congênita, as quais são, na neonatologia, em sua maioria, consideradas urgência cirúrgica. (FRAGA, 2004). Constatou-se que o termo médico teve uma maior freqüência que o termo serviço de enfermagem, também considerado constante nesse eixo, o que leva a crer que a equipe de enfermagem age em muitas situações voltadas para o cumprimento das exigências médicas. De igual forma pode-se comprovar, através dos registros, que algumas ações da equipe dentro da assistência intensiva dependem de ações colaborativas dos médicos.

Discutindo a relevância dos termos compreendidos no eixo AÇÃO destaca-se a alta incidência do termo Evoluir, que apareceu nas suas várias formas de conjugação (evolui - presente do indicativo, evoluindo - gerúndio), mostrando que a evolução do paciente tem uma grande significância para as enfermeiras dentro da assistência prestada, pois é uma condição necessária para avaliar suas ações. MORAIS, CAMPOS \& SILVERSTRINI (2005) referem que a realização de uma assistência contínua ao recém-nascido de alto risco requer um conhecimento detalhado da criança, e, por isso, o prontuário deve ser organizado através da evolução diária do paciente.

Manter foi um termo utilizado para indicar como a assistência na unidade está provendo a necessidade de subsistência do recém-nascido, principalmente, com relação aos utensílios, terapias de suporte utilizadas para manter as condições vitais, como nutrição, temperatura ambiente, respiração. Entende-se sua incidência, principalmente, em uma unidade de terapia intensiva, pois se faz necessária, nessas condições, um controle maior dos suportes das necessidades básicas, levando-se em consideração que o recém-nato está numa fase de adaptação à vida extra-uterina, e, invariavelmente, necessita de algum tipo de aporte vital. Nesse sentido HOCKENBERRY (2006) refere que essa prerrogativa é ocasionada pelo caráter dos pacientes internos em UTIN, que são, em sua maioria, prematuros e/ou de muito baixo peso, e, pelo grau de imaturidade, necessitam, com maior precisão, de manter uma monitorização dos dados fisiológicos, como ambiente térmico controlado, monitorização cardíaca e respiratória.

O termo Aspirar foi encontrado conjugado, principalmente, no pretérito, evidenciando um registro de uma ação muito desempenhada pelas enfermeiras junto ao paciente neonatal que, em sua maioria, necessita de algum nível de terapia respiratória. Ressalta-se essa necessidade pela caracterização desse paciente que está em período de adaptação à vida extra-uterina e atravessa um período de alteração fisiológica de circulação placentária para respiração independente. (HOCKENBERRY, 2006).

Uma grande parte dos neonatos de alto risco, principal população atendida em uma UTIN, irá necessitar de assistência respiratória por períodos variáveis, para estabelecer e manter sua respiração. Dentro dessa perspectiva de condições respiratórias pouco satisfatórias, muitas vezes agravada por presença de secreções nas vias aéreas superiores e inferiores, faz-se necessária a intervenção de profissionais na realização da aspiração, para promover sua recuperação mais facilmente. Essa aspiração torna-se urgente e deve ser realizada de forma mecânica, pelo seu alto nível de eficácia, quando uma quantidade vigorosa e espessa de secreção precisa de ser retirada. (HOCKENBERRY, 2006).

No eixo TEMPO, o termo Presente foi o mais freqüente dentro de um contexto de julgamento de 
presença de sinais durante o exame físico. O Termo Parto foi referido para indicar o tipo de parto ao qual a genitora foi submetida. Essa informação é altamente relevante para se entender o estado de gravidade do recém-nascido, pois, para se prestar uma assistência de alta qualidade ao neonato, deve-se conhecer as condições maternas, pois elas afetam diretamente o feto, e, assim, podem-se antecipar medidas profiláticas ou de tratamento. (TAMEZ \& SILVA, 2002).

No eixo LOCALIZAÇÃO, o termo abdome foi identificado como o mais freqüente desse eixo, como também, o mais freqüente entre todos os outros termos. Infere-se que essa incidência dá-se pela alta ocorrência, entre os recém-nascidos, de algum distúrbio gastrintestinal, seja pela dificuldade de digestão, constipação, pelas infecções intestinais, malformações. Essa observação faz-se salutar para a realização do exame físico do aparelho gastrintestinal, quando nele observam-se todos os seus aspectos, físicos e sonoros, para que esses sinais nos dêem suporte para uma assistência individualizada e de melhor qualidade. (MORAIS; CAMPOS \& SILVERSTRINI, 2005; HOCKENBERRY, 2006; GAÍVA \& GOMES, 2003).

Outro termo bastante encontrado nesse eixo foi Pele, relacionado com algum julgamento, indicando as condições desse órgão tão relevante para um recém-nascido. Na neonatologia a pele é tratada com cuidados especiais, pois, principalmente em prematuros, ela é muito fina, sendo propensa a sofrer lesões, o que pode contribuir para desequilíbrios hidroeletrolíticos e térmicos por perda de água e calor. (TAMEZ \& SILVA, 2002).

Periférico foi um termo encontrado principalmente no julgamento do acesso venoso para indicar a localização mais exata de sua inserção, e, algumas vezes, a presença de cianose do leito ungueal e labial. Nessa mesma perspectiva de direcionar o encontro do local citado, o termo superior foi encontrado para se referir à localização dos membros do corpo, das vias aéreas, dos quadrantes do abdome.

No eixo CLIENTE, encontrou-se o termo recém-nascido que segundo FERREIRA (1999, p.1195), significa "aquele que nasceu há pouco", ou seja, "indivíduo" (CIE, 2006, p.172) compreendido entre 0 a 28 dias de vida. (GAÍVA \& GOMES, 2003). Esse termo foi $o$ mais freqüente neste eixo $e$ evidencia que esse é o sujeito a quem se refere todo o registro de enfermagem, sendo o receptor das ações de enfermagem aqui ressaltadas.

\section{CONSIDERAÇÕES FINAIS}

De acordo com os resultados obtidos, é possível afirmar que os objetivos que nortearam esta pesquisa foram alcançados e que a trajetória metodológica utilizada na elaboração deste trabalho foi adequada o que possibilitou a análise dos resultados e as conclusões finais.

Analisando os dados dos registros, percebe-se que a linguagem comum habita consideravelmente os dados da documentação de enfermagem, mostrando que não existe o emprego formal de uma terminologia da área, e que, apesar dos registros se apresentarem em número bastante relevante, com dados substanciais registrados, a preocupação em tornar a linguagem universal não se mostrou presente, o que nos faz recordar uma proposição de McCORMICK \& JONES (1998), quando referem que, enquanto os praticantes da enfermagem não reconhecerem essa premissa, o vocabulário comum habitará entre as fontes de dados.

É importante que os dados deste estudo estejam precisos e uniformes para serem automatizados e possam servir para a elaboração de um banco de dados apropriado à elaboração de um catálogo aplicável a um sistema de informação voltado para a assistência neonatal. Esse fato seria de alto impacto sobre a assistência de enfermagem prestada na unidade de terapia intensiva neonatal, pois proporcionaria automação do registro de enfermagem o que traria visibilidade para suas ações, auxiliaria a avaliação das mesmas e, subsequentemente, a construção de um corpo de conhecimentos de enfermagem especializados em neonatologia.

Busca-se na assistência de enfermagem em unidade de terapia intensiva neonatal, pela sua alta complexidade em atendimentos, implantar sistemas de referência e hierarquização, pois a assistência nessas unidades, para ser efetiva, necessita de recursos físicos (instalações, equipamentos e insumos) e humanos (médicos, profissionais de enfermagem, fisioterapeutas) muito especializados, o que requer altos investimentos, a necessidade de uma prática crítica, atualização constante e rotinas organizacionais muito estabilizadas.

A realização deste estudo permitiu o conhecimento do vocabulário utilizado no cuidado intensivo ao neonato, entendendo esse tipo de atenção como peculiar, por trata-se de pacientes em período de adaptação à vida extra-uterina, caracterizados com maior vulnerabilidade a morbimortalidade. Dessa forma, a contribuição do mesmo para a futura construção de uma nomenclatura específica para essa clientela é evidente, considerando que a construção de vocabulários universais dá-se sobre conhecimentos e informações obtidas em nível do serviço, refletindo o nível mais alto de integração e síntese de conhecimento, desde que os dados sejam confiáveis, uniformes e precisos (McCORMICK \& JONES, 1998).

Os resultados do estudo confirmam as conjeturas feitas sobre a utilização de linguagens uniformizadas em registros de enfermagem, pois se verifica nos resultados, a alta incidência de termos não constantes na Classificação Internacional para 
Prática de Enfermagem, que tem o intuito de unificar internacionalmente a linguagem utilizada pelos enfermeiros para registrar os fenômenos e ações vinculadas a sua prática, o que confirma a utilização de uma linguagem comum nos registros de enfermagem.

Diante dessa problemática considera-se imprescindível a ênfase no ensino da Graduação de enfermagem sobre essa temática, explorando-se os conhecimentos sobre documentação, linguagens especializadas e terminologias de enfermagem, para que os futuros profissionais da área tenham uma formação condizente com as necessidades vigentes da enfermagem atual.

Espera-se, do mesmo modo, que esses resultados possam expandir-se para aplicação, na extensão, em outras unidades de terapia intensiva de outros hospitais, ou seja, que esse vocabulário identificado possa ser referência, por representar o vocabulário de enfermagem utilizado na terapia intensiva neonatal, e, assim, possa contribuir para a uniformização dos registros e assistência da especialidade.

Almeja-se que, através dos resultados da pesquisa e das considerações feitas, haja a continuação deste estudo em projetos de pesquisa, num futuro próximo. Que sejam realizadas pesquisas para validação dos termos identificados como constantes na $\mathrm{CIPE}^{\circledR}$, como também a análise de inclusão dos termos considerados não constantes nesta classificação, com o objetivo de corroborar os resultados desta pesquisa, e, principalmente, contribuir com a construção da linguagem especializada da área.

\section{REFERÊNCIAS BIBLIOGRÁFICAS}

BITTENCOURT, G.K.G.D. et al. Identificação de fenômenos de enfermagem nos registros dos componentes da equipe de enfermagem na Clínica Médica. In: CONGRESSO BRASILEIRO DE ENFERMAGEM, 54, 2002, Fortaleza - CE. Anais... Fortaleza: Associação Brasileira de Enfermagem, 2002.

BRASIL. Ministério da Saúde. Conselho Nacional de Saúde. Comissão Nacional de Ética em Pesquisa CONEP. Resolução n.196/96 sobre pesquisa envolvendo seres humanos. Brasília, 1996.

CAMPEDELLI, M.C. et al. Processo de Enfermagem na Prática. São Paulo: Ática, 1992.

CAPRA, F. O ponto de mutação - A Ciência, a sociedade e a cultura emergente. 22ed. São Paulo: Ed. Cultrix, 2001.

CARVALHO, W.; CASTRO, I.B. Reflexões sobre a prática da enfermagem. In: Congresso Brasileiro de Enfermagem, 31ํㅡㄹ Fortaleza, 1979. Anais. Brasília, ABEn, 1979. p.51-9.

CONSELHO INTERNACIONAL DE ENFERMAGEM. $\mathrm{CIPE}^{\circledR}$ Versão 1.0. Classificação Internacional para a
Prática de Enfermagem. Lisboa, 2006. Traduzido e publicado com autorização do Conselho Internacional de Enfermeiros, Genebra, Suíça, 2006.

CRUZ, D.A.L.M. Contribuições do diagnóstico de enfermagem para a autonomia da enfermeira. Brasília, DF: Associação Brasileira de Enfermagem, 1997. p.51-57. (Série didática, Enfermagem no SUS). FERREIRA, A.B.H. Dicionário Aurélio Eletrônico Século XXI. Editora Nova Fronteira,1999.

FRAGA, J.C. Urgências cirúrgicas no recém-nascido - detecção e conduta. In: Programa de atualização em Neonatologia - PRORN. Ciclo 2. Módulo 3. Porto Alegre: Artmed, 2004.

GAIIVA, M.A.M.; GOMES, M.M.F. Cuidando do neonato: uma abordagem de enfermagem. Goiânia: $A B, 2003$.

GARCIA, T.R.; NÓBREGA, M.M.L. Sistematização da Assistência de Enfermagem: reflexões sobre o processo. In: CONGRESSO BRASILEIRO DE ENFERMAGEM, 52, 2000, Olinda-PE. Anais... Recife: Associação Brasileira de Enfermagem, 2002. v.1, p.231-243.

GUINSBURG, R.; ALMEIDA, M.F.B. Atualizações 2006 para o manual do Curso de Reanimação Neonatal Documento científico do Programa de Reanimação Neonatal. Reanimação Neonatal: condutas 2006. São Paulo. [online] Sociedade Brasileira de Pediatria. Disponível em: $<$ http://www.sbp.com.br/show item2.cfm?id categoria $=24 \&$ id detalhe $=421 \&$ tipo detalhe $=\mathrm{s}>$. [Acesso em 14 de dez. 2006].

HOCKENBERRY, M.J. Fundamentos de Enfermagem Pediátrica. Rio de Janeiro: Elsevier, 2006.

KARKKAINEN, O.; ERIKSSON.K. Evaluation of patient records as part of developing a nursing care classification. Journal of Clinical Nursing. v.12, n.2, p.198-205, 2003.

KENNER, C. Enfermagem Neonatal. Rio de Janeiro: Reichmann \& Affonso, 2001.

MARIN, H.F. Vocabulários em Enfermagem: Revisão e Atualização. Revista Nursing, v.32, n.1, p.25-29, 2001.

MARIN, M.J.S. et al. Análise das publicações sobre diagnósticos de enfermagem no Brasil. Revista Nursing, v.76, n.7, p23-28, 2004.

McCORMICK, K.A.; JONES, C.B. Is one taxonomy needed for health care vocabularies and classifications? [online] Journal of Issues in Nursing, $1998 . \quad$ Disponível em: $<$ http://nursingworld.org/ojin/tpc7/tpc7 2.htm>

[Acesso em 05 de ago. 2006].

MORAIS, M.B.; CAMPOS, S.O.; SILVERSTRINI, W.S. Guia de Pediatria. Guias de medicina ambulatorial e hospitalar. Barueri, SP: Manole, 2005 NÓBREGA, M.M.L. et al. Mapeamento de termos atribuídos aos fenômenos de enfermagem nos registros dos componentes da equipe de enfermagem. [online] Revista Eletrônica de Enfermagem, v.5, n.2, p.33-44, 2003. Disponível em: 
<http://www.fen.ufg.br/revista/revista5 2/mapa.html> [Acesso em 16 out. 2005]

NÓBREGA, M.M.L.; GARCIA, T.R. Linguagem

Especial da Enfermagem e a Prática Profissional. João Pessoa, 2000. (mimeo).

NÓBREGA, M.M.L.; GARCIA, T.R. Perspectivas de incorporação da Classificação Internacional para a Prática de Enfermagem (CIPE) no Brasil. Revista Brasileira de Enfermagem, v.58, n.2, p.227-230, 2005. PAVEL, S.; NOLET, D. Manual de terminología. Public Works and Government Services: Canadá, 2002.

POSSARI, J.F. SAE: Sistematização da assistência de enfermagem. [online] 2005. Disponível em: <http://www.joaopossari.hpg.ig.com.br/sae.htm>

[Acesso em 17 de ago. 2005].

TAMEZ, R.N.; SILVA, M.J.P. A Enfermagem na UTI Neonatal - Assistência ao recém-nascido de alto risco. 2 ed. Rio de Janeiro: Guanabara Koogan, 2002. TOMA, E. Acesso venoso em recém-nascidos. In: Programa de atualização em Neonatologia - PRORN. Ciclo 3. Módulo 2. Porto Alegre: Artmed, 2004.

VIRGÍNIO, N.A.; NÓBREGA, M.M.L. Sistematização da Assistência de Enfermagem: revisão de literatura. Revista de Saúde Nova Esperança, v.2, n.1, p.15-18, 2004. 